\title{
Interfacial free energy of a hard-sphere fluid in contact with curved hard surfaces
}

\author{
Brian B. Laird, ${ }^{1, *}$ Allie Hunter, ${ }^{1}$ and Ruslan L. Davidchack ${ }^{2}$ \\ ${ }^{1}$ Department of Chemistry, University of Kansas, Lawrence, Kansas 66045, USA \\ ${ }^{2}$ Department of Mathematics, University of Leicester, Leicester, LE1 7RH, United Kingdom
}

(Received 7 May 2012; published 20 December 2012)

\begin{abstract}
Using molecular-dynamics simulation, we have calculated the interfacial free energy $\gamma$ between a hard-sphere fluid and hard spherical and cylindrical colloidal particles, as functions of the particle radius $R$ and the fluid packing fraction $\eta=\rho \sigma^{3} / 6$, where $\rho$ and $\sigma$ are the number density and hard-sphere diameter, respectively. These results verify that Hadwiger's theorem from integral geometry, which predicts that $\gamma$ for a fluid at a surface, with certain restrictions, should be a linear combination of the average mean and Gaussian surface curvatures, is valid within the precision of the calculation for spherical and cylindrical surfaces up to $\eta \approx 0.42$. In addition, earlier results for $\gamma$ for this system [Bryk et al., Phys. Rev. E 68, 031602 (2003)] using a geometrically based classical density functional theory are in excellent agreement with the current simulation results for packing fractions in the range where Hadwiger's theorem is valid. However, above $\eta \approx 0.42, \gamma(R)$ shows significant deviations from the Hadwiger form indicating limitations to its use for high-density hard-sphere fluids. Using the results of this study together with Hadwiger's theorem allows one, in principle, to determine $\gamma$ for any sufficiently smooth surface immersed in a hard-sphere fluid.
\end{abstract}

DOI: 10.1103/PhysRevE.86.060602

PACS number(s): 68.08.De, 64.70.D-, 05.70.Np, 68.35.Md

The solid-liquid interfacial free energy $\gamma$ is a central property governing a wide variety of technologically important phenomena from crystal nucleation and growth to wetting. Because accurate and reliable experimental measurements of $\gamma$ are rare, much effort has been devoted in recent years to the development of atomistic simulation methods to determine this quantity for interfaces between coexisting solid and fluid phases [1-5] and for systems in which the solid is modeled by a static wall [6-11]. These efforts have thus far been primarily restricted to planar interfaces; however, there are many physically relevant systems in which interfacial curvature is relevant, for example, in the formation of critical nuclei in nucleation [12-14] or the solvation or wetting of hydrophobic nanoscale particles [15-17]. There have been a number of previous simulation studies that examine the effect of curvature in liquid-vapor interfaces [18-21], but direct simulation studies on solid-liquid interfaces are lacking. In this Rapid Communication, we examine the dependence of $\gamma$ on the surface curvature for a hard-sphere fluid in contact with curved hard surfaces, specifically at spherical and cylindrical colloidal particles.

König et al. [22] have recently shown that Hadwiger's theorem [23] from integral geometry puts severe restrictions on the shape (curvature) dependence of the interfacial free energy. In their analysis, the interfacial free energy of an object with a surface $S$ is given by

$$
\gamma(S)=\gamma_{0}+h \bar{H}+\kappa \bar{K},
$$

where $h$ and $\kappa$ are constants depending upon the thermodynamic state, but independent of the specific surface $S$. Here $\bar{H}$ and $\bar{K}$ are the averaged mean and Gaussian curvatures of $S$,

\footnotetext{
*Author to whom correspondence should be addressed: blaird@ku.edu
}

defined as

$$
\begin{gathered}
\bar{H}=\frac{1}{2 A} \int_{S}\left[\frac{1}{R_{1}(\vec{q})}+\frac{1}{R_{2}(\vec{q})}\right] d S, \\
\bar{K}=\frac{1}{A} \int_{S}\left[\frac{1}{R_{1}(\vec{q}) \cdot R_{2}(\vec{q})}\right] d S,
\end{gathered}
$$

where $R_{1}(\vec{q})$ and $R_{2}(\vec{q})$ are the principal radii of curvature at each point $\vec{q}$ on the surface $S$, and $A$ is the surface area. As discussed in Ref. [22], the use of Hadwiger's theorem to determine the free energy of fluids at bounding surfaces, often referred to as "morphological thermodynamics," is valid as long as the fluid-surface system satisfies motion invariance, continuity, and additivity. These conditions can break down for situations in which the bounding surface is small (i.e., on the order of the fluid particle size), for systems with long-range interactions, or for highly concave surfaces in which the fluid is confined in regions smaller than a few correlation lengths. For a fluid in contact with a spherical convex surface of radius $R, \bar{H}_{s}=1 / R$ and $\bar{K}_{s}=1 / R^{2}$, while for a fluid in contact with a convex cylinder of the same radius, one has $\bar{H}_{c}=1 / 2 R$ and $\bar{K}_{c}=0$. Much of the previous work on the curvature dependence focused on the first-order curvature correction [24], referred to as the Tolman length $\delta$ which, for a convex spherical surface, would be given in terms of $h$ by $\delta \gamma_{0}=-h / 2$.

The first theoretical treatment for the curvature dependence of $\gamma$ for a hard-sphere fluid at a hard wall was developed within the so-called scaled particle theory (SPT) [16,25,26], which is a theory of solvation that is based on an approximate determination of the work required to insert a spherical cavity (or hard-sphere solute) into a fluid. For this system, $\gamma$ scales trivially with $T$, that is, $\gamma(\eta, T)=\gamma^{*}(\eta) k T / \sigma^{2}$, where $\gamma^{*}$ is the reduced interfacial free energy, $k$ is Boltzmann's constant, $T$ is temperature, and $\sigma$ is the hard-sphere diameter of the fluid. The SPT result for $\gamma^{*}$ for a hard-sphere fluid at a convex 
spherical surface of radius $R$ is given by

$$
\gamma_{\mathrm{SPT}}^{*}(\eta)=\frac{3 \eta(2+\eta)}{2 \pi(1-\eta)^{2}}+\frac{3 \eta}{2 \pi(1-\eta)} \frac{1}{R}-\frac{\ln (1-\eta)}{4 \pi} \frac{1}{R^{2}},
$$

where $\eta$ is the packing fraction, defined in terms of the particle density $\rho$ as $\eta=\pi \rho \sigma^{3} / 6$. (For simplicity in what follows we measure distance in units of $\sigma$ and energy in units of $k T$ and drop the * superscript for reduced units.) The SPT treatment is consistent with the Hadwiger form of $\gamma$ [Eq. (1)] with $h$ and $\kappa$ equal to the coefficients of $1 / R$ and $1 / R^{2}$ in Eq. (4), respectively. Note that the value of the interfacial free energy between a fluid and a wall depends upon the precise definition of the dividing surface that determines the volume of the bounding wall. In this work, we define the dividing surface to be coincident with the wall surface.

Using classical density functional theory (DFT), Bryk et al. [27] have examined the scaling of the interfacial free energy and the excess interfacial adsorption for hard-sphere fluid at both spherical and cylindrical hard surfaces. This work, which utilized the fundamental measure theory (FMT) version of DFT proposed by Rosenfeld [28], determined $\gamma$ for this system as a function of packing fraction and curvature with $1 / R$ ranging from 0 to 0.5 . The results for the spherical and cylindrical geometries were fit to a polynomial of the form $\gamma(R)=\gamma_{0}+a_{1} / R+a_{2} / R^{2}$ and were consistent with Hadwiger's theorem in that the ratio of $a_{1}$ for the spherical surface to that of the cylinder was 2 . The predicted FMT values for $h$ for both geometries are in good agreement with the SPT results [Eq. (4)] at low packing fractions, but exhibit a negative deviation from the SPT curve of a few percent at the higher densities studied. More recently, Jin et al. [17] examined the solvation free energy of various ideal nanoscale nonspherical shapes (for example, cones, cylinders, and prisms) using a hybrid Monte Carlo DFT technique. They conclude that the use of "morphological thermodynamics" based on Hadwiger's theorem to determine the solvation free energy (which includes both bulk and interfacial free energy components) for the nonspherical particles compares well with the DFT results. To date, there have been no determinations of $\gamma$ from direct simulation for the hard-sphere fluid at curved surfaces with which to assess the accuracy of these DFT results. This is the principal goal of the present study.

In this work, we make use of an adsorption equation derived using Cahn's extension [29] of the surface thermodynamics of Gibbs [30], namely,

$$
\left(\frac{\partial \gamma}{\partial P}\right)_{T}=v_{N}
$$

where the excess interfacial volume per unit area $v_{N}$ is defined by

$$
v_{N}=\frac{1}{A N_{f}}\left|\begin{array}{cc}
V & N \\
V_{f} & N_{f}
\end{array}\right|=\frac{1}{A}\left(V-V_{f} \frac{N}{N_{f}}\right),
$$

where $A$ is the interfacial area, $V$ and $N$ are the volume and number of particles, respectively, of a region containing the interface, and $V_{f}$ and $N_{f}$ are the corresponding quantities for a region entirely within the bulk fluid [11]. This adsorption equation can be shown to be equivalent through a change of variables and Maxwell relations to the usual Gibbs adsorption equation

$$
\left(\frac{\partial \gamma}{\partial \mu}\right)_{T}=\Gamma_{N}
$$

where $\Gamma_{N}$ is the excess interfacial number of particles per unit area at the interface. Equation (5) can be integrated with respect to pressure to give

$$
\gamma(P)=\gamma_{P=0}+\int_{0}^{P} v_{N}(P) d P,
$$

where $P$ is the pressure. For the hard-sphere fluid at the hardwall, $\gamma_{P=0}=0$. Equation (7) was recently used to determine $\gamma$ for a hard-sphere fluid at a flat wall [11]. The excess interfacial volume can be related to the density profile $\rho(r)$ by

$$
v_{N}=\frac{1}{R^{i}} \int_{R}^{\infty}\left[1-\frac{\rho(r)}{\rho_{f}}\right] r^{i} d r
$$

where $i=1$ and 2 for the cylindrical and spherical geometries, respectively.

To determine $\gamma$ for this system using Eq. (7), we use molecular-dynamics (MD) simulation to calculate $v_{N}$ as a function of bulk packing fraction $\eta=\rho \sigma^{3} / 6$ for a hardsphere fluid in contact with spherical and cylindrical colloidal particles of radius $R$ varying from 10 down to 0.5 , over a range of $\eta$ from 0.03 to 0.49 , the upper limit being near the fluid packing fraction at freezing. For the simulations, we use the algorithm of Rapaport [31] and use Eq. (6) directly to determine $v_{N}$ in the simulations. For additional simulation details and plots of $v_{N}(\eta ; R)$ see the Supplemental Material [32].

Because we find $v_{N}$ as a function of the packing fraction, not the pressure, we transform Eq. (7) to give

$$
\gamma(\eta)=\int_{0}^{\eta} v_{N}\left(\eta^{\prime}\right)\left(\frac{\partial P}{\partial \eta^{\prime}}\right)_{T} d \eta^{\prime} .
$$

To obtain the derivative of $P$ with respect to $\eta$ we use the Kolafa, Labík, and Malijevský (KLM)-low equation of state (EOS) [33]. This EOS has been shown to give five decimal place accuracy in the pressure even at high density when compared to high-quality simulations [34], so any errors introduced by its use are much smaller than the statistical error in the simulation data. To reduce the numerical integration error in evaluating Eq. (9) we subtract from the integrand the corresponding value obtained from the SPT [Eq. (4)]-the corresponding SPT expression for the cylindrical surface is obtained from Eq. (4) by setting the coefficient of the $1 / R^{2}$ term to zero and dividing the coefficient of the $1 / R$ term by two. Accurate calculation of the excess interfacial volume $v_{N}$ at very low densities is difficult because of sampling issues; however, this quantity can be calculated exactly in the limit $\rho \rightarrow 0$. In this limit, we have

$$
\lim _{\rho \rightarrow 0} v_{N}(R)=\frac{1}{2}+\frac{a_{1}}{R}+\frac{a_{2}}{R^{2}},
$$

where $a_{1}$ and $a_{2}$ are constants equal to $1 / 4(1 / 8)$ and $1 / 24(0)$, respectively, for the spherical (cylindrical) wall.

For small values of $R$, the statistical errors in $v_{N}$ are larger for similar simulation lengths because the number of particles near the wall is relatively small. For the special case 
of $R=0.5$ where the spherical surface is identical in size to the fluid particles, high precision results can be generated by recognizing that the density profile $\rho(r)$ is equivalent to $\rho_{f} g(r)$, where $g(r)$ is the radial distribution in a bulk hard-sphere fluid with density $\rho_{f}$. For this value of $R$, we use an MD simulation for a bulk hard-sphere fluid to calculate $g(r)$ from which $v_{N}$ can be determined with high precision. Alternatively, by replacing $\rho(r)$ with $\rho_{f} g(r)$ for $R=0.5$, we have from Eq. (8) that

$$
\begin{aligned}
v_{N}(R=0.5) & =4 \int_{1 / 2}^{\infty} r^{2}[1-g(r)] d r \\
& =-\frac{1}{\pi} \int_{0}^{\infty} 4 \pi r^{2}[g(r)-1] d r-1 / 6 .
\end{aligned}
$$

Using the compressibility equation from liquid state physics [35], the integral in the previous equation can be replaced with a term dependent upon the isothermal compressibility $\kappa_{T}=\rho^{-1}(\partial \rho / \partial P)_{T}$ to yield

$$
v_{N}(\rho ; R=0.5)=-\frac{1}{\pi}\left(k T \kappa_{T}-\rho^{-1}\right)-1 / 6 .
$$

Using an equation of state, the isothermal compressibility $\kappa_{T}$ can be determined analytically as a function of $\eta$ allowing for an analytical calculation of $v_{N}$, and thus $\gamma$ for $R=0.5$.

The calculated values of $\gamma$ from our simulations for the spherical and cylindrical walls are plotted as functions of $\eta$ in Fig. 1, for values of $R$ ranging from the planar wall $(R=\infty)$ to 0.5 . At all densities, these figures show that at fixed $\eta$, the interfacial free energy $\gamma$ is a monotonically increasing function of $1 / R$.

To test the validity of Hadwiger's theorem for this system, we fit the data shown in Fig. 1 to Eq. (1) to determine the constants $h$ and $\kappa$ for each $\eta$. The fit was performed using standard weighted quadratic (for the spherical case where $\bar{H}=$ $1 / R$ and $\bar{K}=1 / R^{2}$ ) and linear (for the cylindrical case where

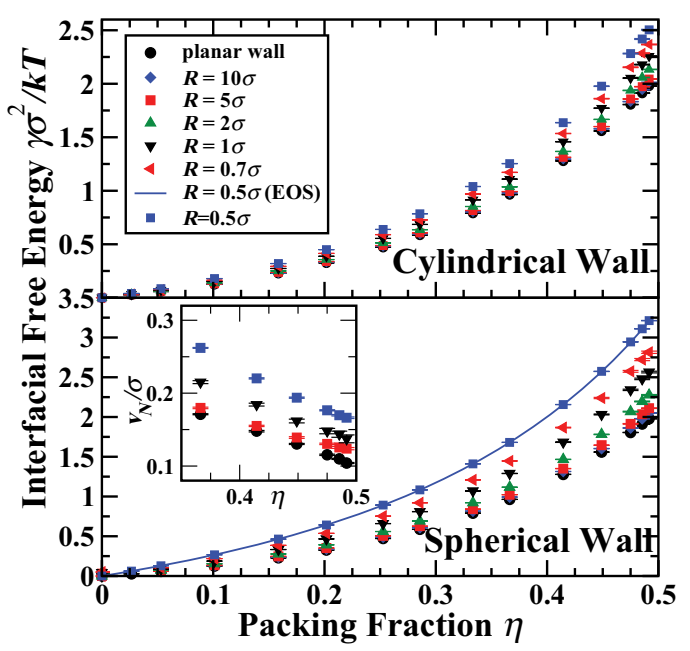

FIG. 1. (Color online) Top: Interfacial free energy between a hard-sphere fluid and a hard cylindrical colloidal particle as a function of $\eta$ for several values of the radius $R$. Bottom: Same as the top panel except for the spherical surface. The inset shows the value of the excess volume $v_{N}$ for the spherical surface as a function of packing fraction for $R=\infty, 5,1$, and 0.5 . The symbols in this panel and in the inset are as indicated in the top panel legend.

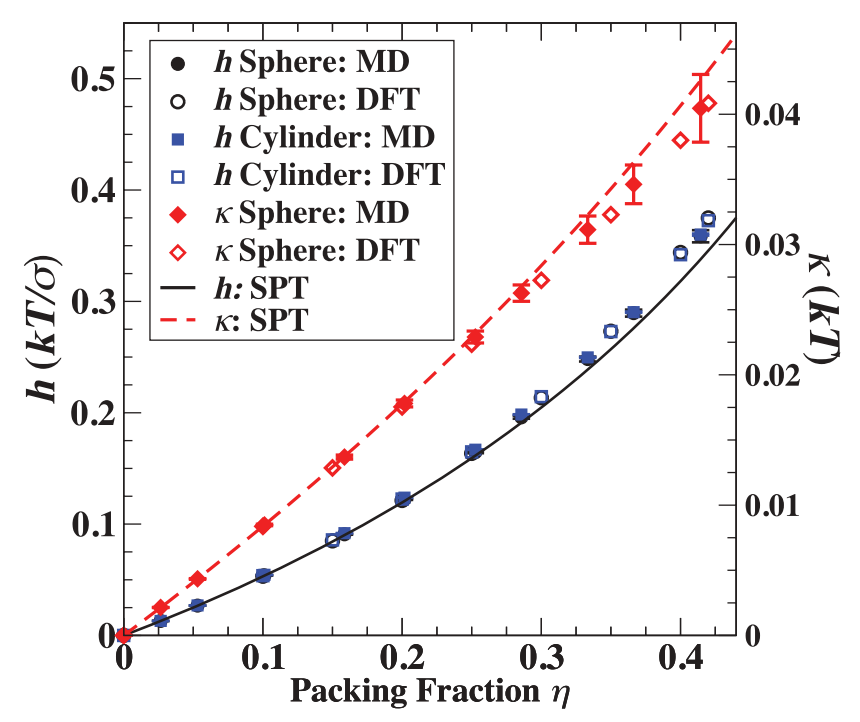

FIG. 2. (Color online) The calculated values of $h$ and $\kappa$ for the spherical and cylindrical walls. Solid symbols are the results from the present MD simulations and the open symbols are the values calculated from DFT [27]. The solid and dashed lines are the values of $h$ and $\kappa$ from SPT [Eq. (4)]. Note that the symbols for the various values of $h$ are difficult to resolve from one another because they are nearly coincident.

$\bar{H}=1 / 2 R$ and $\bar{K}=0$ ) least-squares regression. The weights in the regression were equal to the inverse of the statistical variance of the data points. The results of these fits are shown in Fig. 2 for packing fractions up to 0.42. A table of the fitted values for $\gamma_{0}, h$ and $\kappa$ is included in the Supplemental Material [32]. Also, shown in Fig. 2 are the results for $h$ and $\kappa$ from the SPT expression (4) and from the Rosenfeld DFT [27]. The DFT results agree remarkably well with the simulation results for the values of $\eta$ shown in Fig. 2. Most notable in these results is the fact that the estimates for $h$ for the spherical and cylindrical geometries shown in Fig. 2 are in excellent agreement, as predicted by Hadwiger's theorem. The SPT prediction agrees well with the simulation results at low packing fractions, but underestimates $h$ and slightly overestimates $\kappa$ at packing fractions above about 0.25 . Note that, as mentioned earlier, the value of $\gamma$ and, by extension, the Hadwiger coefficients $\gamma_{0}, h$, and $\kappa$ will depend upon the choice of dividing surface; however, the Hadwiger form [Eq. (1)] remains valid, albeit with modified coefficients [16].

Above $\eta=0.42$, however, significant deviations from the Hadwiger form were found. To quantify these deviations, we performed a cubic and quadratic weighted least-squares regression with respect to $1 / R$ on the sphere and cylinder data, respectively. The results of these calculations are plotted in Fig. 3. At packing fractions below 0.42 , the cubic coefficient $\left(a_{3}\right)$ for the spherical surface is zero within the estimated statistical error, consistent with Hadwiger's theorem, but this coefficient diverges quickly from zero at packing fractions above 0.42 , indicating a significant breakdown of the Hadwiger form at packing fractions approaching the freezing density $(\eta=0.492)$. The situation is similar for the quadratic coefficient $\left(a_{2}\right)$ for the cylindrical case (Fig. 3, top panel) - there is one value of $a_{2}$ at $\eta \approx 0.25$ that is marginally different from 


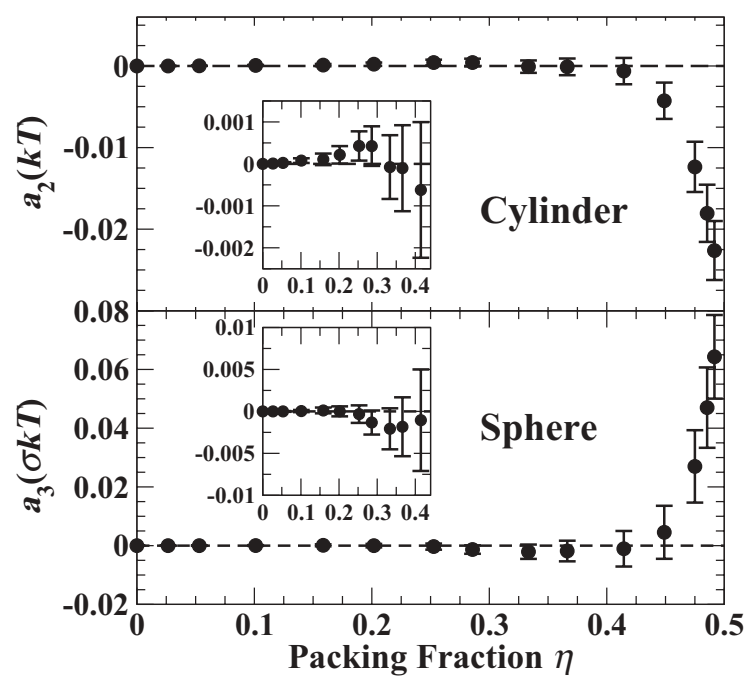

FIG. 3. Top: Plot of the first non-Hadwiger coefficient $a_{2}(\eta)$ in the weighted least-squares fit to a quadratic polynomial in $1 / \mathrm{R}$ of $\gamma$ for the hard-sphere fluid at a cylindrical colloidal particle. Bottom: Same as the top panel, but for $a_{3}(\eta)$ in the cubic polynomial fit of $\gamma$ at a spherical colloidal particle. For clarify, the insets show the data for $\eta<0.42$ on a smaller scale.

zero outside the error bars, but given its marginality and the small value of the coefficient, we do not view this deviation as significant. The origin of the divergence of the non-Hadwiger coefficients at high packing fraction can be seen in the inset in Fig. 1 in which the excess volume $v_{N}$ is plotted for the spherical surface as a function of $\eta$ for $R=\infty, 5,1$, and 0.5 . At high $\eta$, the excess volume $v_{N}$ for the planar wall $(R=\infty)$ exhibits a downward curvature that is not present in the other values of $R$, making it impossible to fit $v_{N}(R)$ purely as a quadratic polynomial in $1 / R$. It is possible that this anomalous decrease in $v_{N}$ for the planar wall is connected to the subcritical fluctuations associated with the prefreezing transition that has been observed for the hard-sphere fluid at a hard planar wall at high packing fraction [10,36,37], and further analysis is underway.

These results also indicate that the errors in the SPT at the intermediate packing fractions are not likely to be due to the omission of higher-order terms (beyond the quadratic for the sphere) in $1 / R$, as has been proposed (see Ref. [38] for a review). The results here put severe upper bounds on the magnitude of any such higher-order terms. As an example, our calculated value for $\gamma$ at $\eta=0.25265$ and $R=1.0$ is $0.6595(6)$. The corresponding SPT value from Eq. (4) is 0.6711 - a difference of $0.0116(6)$, which is more than an order of magnitude larger than the estimated upper bound to the contribution of the cubic term $a_{3}$ at this packing fraction (see Fig. 3). Note that the largest contribution to the difference comes from $\gamma_{0}$, the value for the planar wall.

In summary, we have calculated the curvature dependence of the interfacial free energy between hard-sphere fluid and hard spherical and cylindrical colloidal particles of varying radius $R$. These calculations have important applications in determining the solvation free energy of nanoscale objects. From our simulation results, we have verified that the predictions of Hadwiger's theorem [22,23], which predicts that $\gamma$ for fluids at a bounding surface, under certain restrictions, is completely described by a linear combination of the average mean and Gaussian curvatures, are valid for the systems studied at low to moderate fluid packing fractions $(\eta<0.42)$ and have calculated the coefficients of this linear combination ( $h$ and $\kappa$ ) as functions $\eta$. For these packing fractions, the Hadwiger form for $\gamma$ [Eq. (1)] is shown to be valid for both convex spherical and cylindrical surfaces even down to $R=0.5$, where the radius of the bounding surface is equal to that of the fluid particles. In addition, earlier DFT results based on Rosenfeld's fundamental measure theory are found to be in excellent agreement with the results of the current simulations. At higher packing fractions $(\eta>0.42)$, however, significant deviations from the Hadwiger form are observed. The results of these simulations can serve as a useful reference model to determine the thermodynamics of the hydrophobic solvation of nanoscale particles.

B.B.L. acknowledges support from the National Science Foundation (NSF) under Grant No. CHE-0957102. We also acknowledge the KU Research Experiences for Undergraduates funded by NSF under Grant No. CHE-1004897 for providing A.H. with support to work on this project. R.L.D. did part of the work during a study leave granted by the University of Leicester. This research used the ALICE High Performance Computing Facility at the University of Leicester. We also wish to thank Dr. Roland Roth for helpful discussions.
[1] J. Q. Broughton and G. H. Gilmer, J. Chem. Phys. 84, 5759 (1986).

[2] R. L. Davidchack and B. B. Laird, Phys. Rev. Lett. 85, 4751 (2000).

[3] J. J. Hoyt, M. Asta, and A. Karma, Phys. Rev. Lett. 86, 5530 (2001).

[4] B. B. Laird and R. L. Davidchack, J. Phys. Chem. B 109, 17802 (2005).

[5] X. Feng and B. B. Laird, J. Chem. Phys. 124, 044707 (2006).

[6] M. Heni and H. Löwen, Phys. Rev. E 60, 7057 (1999).

[7] E. De Miguel and G. Jackson, Mol. Phys. 104, 3717 (2006).
[8] A. Fortini and M. Dijkstra, J. Phys.: Condens. Matter 18, L371 (2006)

[9] A. Fortini, Ph.D. thesis, Utrecht University, 2007.

[10] B. B. Laird and R. L. Davidchack, J. Phys. Chem. C 111, 15952 (2007).

[11] B. B. Laird and R. L. Davidchack, J. Chem. Phys. 132, 204101 (2010).

[12] A. Cacciuto, S. Auer, and D. Frenkel, J. Chem. Phys. 119, 7467 (2003).

[13] D. T. Wu, L. Granasy, and F. Spaepen, MRS Bull. 29, 945 (2004).

[14] X.-M. Bai and M. Li, J. Chem. Phys. 124, 124707 (2006). 
[15] K. Lum, D. Chandler, and J. D. Weeks, J. Phys. Chem. B 103, 4570 (1999).

[16] J. R. Henderson, J. Chem. Phys. 116, 5039 (2002).

[17] Z. Jin, J. Kim, and J. Wu, Langmuir 28, 6997 (2012).

[18] S. M. Thompson, K. E. Gubbins, J. P. R. B. Walton, R. A. R. Chantry, and J. S. Rowlinson, J. Chem. Phys. 81, 530 (1984).

[19] M. J. P. Nijmeijer, C. Bruin, A. B. van Woerkom, A. F. Bakker, and J. M. J. van Leeuwen, J. Chem. Phys. 96, 565 (1992).

[20] B. J. Block, S. J. Das, M. Oettel, P. Virnau, and K. Binder, J. Chem. Phys. 133, 154702 (2010).

[21] A. Tröster, M. Oettel, B. Block, P. Virnau, and K. Binder, J. Chem. Phys. 136, 064709 (2012).

[22] P. M. König, R. Roth, and K. R. Mecke, Phys. Rev. Lett. 93, 160601 (2004).

[23] H. Hadwiger, Vorlesung über Inhalt Oberfläche und Isoperimetrie (Springer, Berlin, 1957).

[24] R. C. Tolman, J. Chem. Phys. 17, 333 (1949).

[25] H. Reiss, H. L. Frisch, E. Helfand, and J. L. Lebowitz, J. Chem. Phys. 32, 119 (1960).

[26] H. Reiss, Adv. Chem. Phys. 9, 1 (1965).

[27] P. Bryk, R. Roth, K. R. Mecke, and S. Dietrich, Phys. Rev. E 68 , 031602 (2003).
[28] Y. Rosenfeld, Phys. Rev. Lett. 63, 980 (1989).

[29] J. W. Cahn, in Interfacial Segregation, edited by W. C. Johnson and J. M. Blakely (ASM International, International Materials Park, OH, 1979), pp. 3-23.

[30] J. W. Gibbs, The Collected Works, Vol. 1 (Yale University Press, New Haven, 1957).

[31] D. C. Rapaport, The Art of Molecular Dynamics Simulation (Cambridge University Press, New York, 1995).

[32] See Supplemental Material at http://link.aps.org/supplemental/ 10.1103/PhysRevE.86.060602 for additional simulation details and intermediate results.

[33] J. Kolafa, S. Labík, and A. Malijevský, Phys. Chem. Chem. Phys. 6, 2335 (2004).

[34] M. N. Bannerman, L. Lue, and L. V. Woodcock, J. Chem. Phys. 132, 084507 (2010).

[35] J. P. Hansen and I. R. McDonald, Theory of Simple Liquids, 3rd ed. (Academic, New York, 2006).

[36] D. J. Courtemanche and F. van Swol, Phys. Rev. Lett. 69, 2078 (1992)

[37] M. Dijkstra, Phys. Rev. Lett. 93, 108303 (2004).

[38] H. S. Ashbaugh and L. R. Pratt, Rev. Mod. Phys. 78, 159 (2006). 